\title{
Kanakugiol, a Compound Isolated from Lindera erythrocarpa, Promotes Cell Death by Inducing Mitotic Catastrophe after Cell Cycle Arrest ${ }^{\text {s }}$
}

\author{
Jintak Lee ${ }^{1 \dagger}$, Hyun-Woo Chun ${ }^{1 \dagger}$, Thu-Huyen Pham ${ }^{1}$, Jae-Hwan Yoon ${ }^{1}$, Jiyon Lee ${ }^{1}$, Myoung-Kwon Choi ${ }^{1}$, \\ Hyung-Won $\mathrm{Ryu}^{2}$, Sei-Ryang $\mathrm{Oh}^{2}$, Jaewook $\mathrm{Oh}^{3}$, and Do-Young Yoon ${ }^{1 *}$ \\ ${ }^{1}$ Department of Bioscience and Biotechnology, Research Institute of Bioactive-Metabolome Network, Konkuk University, Seoul 05029, \\ Republic of Korea \\ ${ }^{2}$ Natural Medicine Research Center, Korea Research Institute of Bioscience and Biotechnology, Cheongju 28116, Republic of Korea \\ ${ }^{3}$ Department of Stem Cell and Regenerative Biotechnology, Konkuk University, Seoul 05029, Republic of Korea
}

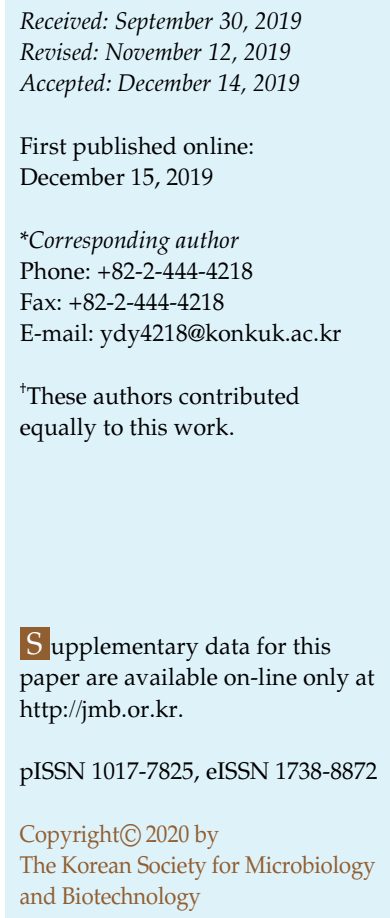

S upplementary data for this paper are available on-line only at http://jmb.or.kr.

pISSN 1017-7825, eISSN 1738-8872

Copyright $(2020$ by

The Korean Society for Microbiology and Biotechnology

\begin{abstract}
A novel compound named 'kanakugiol' was recently isolated from Lindera erythrocarpa and showed free radical-scavenging and antifungal activities. However, the details of the anticancer effect of kanakugiol on breast cancer cells remain unclear. We investigated the effect of kanakugiol on the growth of MCF-7 human breast cancer cells. Kanakugiol affected cell cycle progression, and decreased cell viability in MCF-7 cells in a dose-dependent manner. It also enhanced PARP cleavage (50 kDa), whereas DNA laddering was not induced. FACS analysis with annexin V-FITC/PI staining showed necrosis induction in kanakugiol-treated cells. Caspase-9 cleavage was also induced. Expression of death receptors was not altered. However, Bcl-2 expression was suppressed, and mitochondrial membrane potential collapsed, indicating limited apoptosis induction by kanakugiol. Immunofluorescence analysis using $\alpha-$ tubulin staining revealed mitotic exit without cytokinesis ( $4 \mathrm{~N}$ cells with two nuclei) due to kanakugiol treatment, suggesting that mitotic catastrophe may have been induced via microtubule destabilization. Furthermore, cell cycle analysis results also indicated mitotic catastrophe after cell cycle arrest in MCF-7 cells due to kanakugiol treatment. These findings suggest that kanakugiol inhibits cell proliferation and promotes cell death by inducing mitotic catastrophe after cell cycle arrest. Thus, kanakugiol shows potential for use as a drug in the treatment of human breast cancer.
\end{abstract}

Keywords: Kanakugiol, breast cancer, necrosis, mitotic catastrophe, cell cycle arrest

\section{Introduction}

Cancer is one of the most important threats to human health [1]. All tumor cells share a common characteristic, that of defective or failed mitosis leading to the generation of aneuploid or tetraploid cells [2, 3]. Theodor Boveri postulated more than 100 years ago that abnormal chromosome segregation during mitosis might induce tumor formation [4]. Breast cancer is among the most common causes of cancer-related deaths [5], and the worldwide incidence of breast cancer is highest among women [6]. Although significant progress has recently been achieved in early detection and diagnosis of breast cancer, the incidence of this cancer type remains high [7]. Breast cancers are generally resistant to therapeutics targeting apoptosis due to the status of tumor-suppressing gene products [8]. Therefore, therapies inducing other types of cell death are preferentially used in breast cancer treatment [9].

Various possibilities must be considered during the development of anticancer drugs. For instance, even though apoptosis induction is the most common target of anticancer drugs, some cancers still escape apoptosis by regulating apoptotic signals [10]. Recent advances in our understanding of cancer biology have enabled us to develop new targeting strategies in anticancer drug development 
to suppress tumor growth. Microtubule-stabilizing and -destabilizing agents are the two types of microtubuletargeting antimitotic drugs. Destabilizing agents, such as vinca alkaloids and colchicines, bind to one of the two tubulin classes, and thus prevent microtubule polymerization. However, most stabilizing agents bind to the taxoid binding site of $\beta$-tubulin, and result in enhanced microtubule polymerization [11].

Microtubules are important components of the cytoskeleton that are composed of $\alpha$ - and $\beta$-tubulin subunits, which dimerize to form linear protofilaments. These protofilaments then form the final microtubule structure. The dynamic nature of the microtubule plus ends with continuous polymerization and depolymerization allows them to form the cell structure, while enabling motility and intracellular transport [12]. Therefore, regulation of the microtubule function might be an effective alternative to inducing tumor cell death.

Uncontrolled cell proliferation and apoptosis evasion are typical tumor cell characteristics. Apoptosis is a mechanism of genetically programmed cell death, and is morphologically different from other cell death processes, which can be induced by various physiological and pathological stimuli [13]. Necrosis is an accidental cell death mechanism that generally occurs without activation of any signal transduction process. Interestingly, necrosis was recently shown to also occur through a programmed signaling pathway [14, 15]. Shah et al. demonstrated the presence of the $50 \mathrm{kDa}$ major fragment of PARP in necrotic cell death [16]. Thus, this major fragment of PARP (50 kDa) is a necrotic signature. In 2012, the International Nomenclature Committee on Cell Death defined mitotic catastrophe as an intrinsic tumorsuppressing mechanism that detects mitotic failure, and drives a cell to an irreversible anti-proliferative death [17]. Although it does not directly induce cell death, mitotic catastrophe progresses by triggering anti-proliferative processes including apoptosis and necrosis to inhibit the proliferation of a cell with defective mitosis. Apoptosisand necrosis-related morphological and biochemical characteristics can be observed during mitotic catastrophe due to the adoption of a variety of anti-proliferative pathways [12, 18]. Therefore, investigation of pathways related to apoptosis, necrosis, and mitotic catastrophe is recommended for finding new therapeutic cancer targets.

Kanakugiol, a compound recently isolated from Lindera erythrocarpa, was previously shown to have free radicalscavenging and antifungal activities $[19,20]$. However, the information regarding the effect of kanakugiol on breast cancer cells remains unclear. Thus, there is a rationale for testing the anti-breast cancer effects of kanakugiol. Here, we demonstrate that a small molecule, kanakugiol, promotes cell death in breast cancer by inducing mitotic catastrophe after cell cycle arrest; thus, kanakugiol can be considered as a potential drug for treating breast cancer.

\section{Materials and Methods}

\section{Instruments and Reagents}

1D $\left({ }^{1} \mathrm{H},{ }^{13} \mathrm{C}\right.$, and DEPT) and 2D (COSY, HMQC, and HMBC) NMR spectra were obtained using Bruker AM 400 spectrometers (Bruker, USA) with tetramethylsilane (TMS) as the internal standard. HRESIMS (high-resolution electrospray ionization mass spectrometry) analysis was conducted on an ultraperformance liquid chromatography-quadrupole time-of-flight mass spectrometer (UPLC-QTOF-MS, Waters, USA) in the positive-ion mode.

\section{Extraction and Isolation}

L. erythrocarpa fruits were collected from Jeju Island, Korea during October 2013. Dried fruits $(5.0 \mathrm{~kg})$ were subjected to methanol extraction $(15 \mathrm{~L} \times 2)$ three times at room temperature, to obtain solid extract $(770.0 \mathrm{~g})$. Next, $500 \mathrm{~g}$ of the extract was fractionated using a silica gel column $(10 \times 90 \mathrm{~cm}$, JEO prep 60, $40-63 \mu \mathrm{m}, 2.3 \mathrm{~kg}$ ), and eluted using hexane-EtOAc mixtures $(20: 1 \rightarrow 15: 1 \rightarrow 10: 1 \rightarrow 8: 1 \rightarrow 6: 1 \rightarrow 4: 1 \rightarrow 2: 1 \rightarrow 1: 1)$ to obtain 10 pooled fractions (LE Frs. 1-10). These were then combined based on comparison of their TLC and UPLC-PDA profiles. LE Fr. 4 (105.5 g) with kanakugiol was isolated by column chromatography with reverse phase silica gel $(11 \times 90 \mathrm{~cm}$, Zeoprep C18 $75 \mu \mathrm{m}, 4.0 \mathrm{~kg})$, and eluted using $\mathrm{MeOH} / \mathrm{DW}(40 \% \rightarrow 60 \% \rightarrow 80 \rightarrow 100 \%)$ to isolate kanakugiol (4.3 g). Finally, purified kanakugiol was identified by comparing its MS and NMR spectral data with published literature [19].

\section{Spectroscopic and Physical Data for Kanakugiol}

Yellow oil; UV (MeOH) $\lambda_{\max } \mathrm{nm} \mathrm{208,314;}{ }^{1} \mathrm{H}$ NMR $(400 \mathrm{MHz}$, $\left.\mathrm{CDCl}_{3}\right) \delta 7.91(1 \mathrm{H}, \mathrm{d}, J=15.5 \mathrm{~Hz}, \mathrm{H}-\alpha), 7.82(1 \mathrm{H}, \mathrm{d}, J=15.5 \mathrm{~Hz}, \mathrm{H}-$ ß), $7.62(2 \mathrm{H}, \mathrm{m}, \mathrm{H}-2$ and $\mathrm{H}-6), 7.40(3 \mathrm{H}, \mathrm{m}, \mathrm{H}-3, \mathrm{H}-4, \mathrm{H}-5), 4.08$ $\left(3 \mathrm{H}, \mathrm{s}, 2^{\prime}-\mathrm{OCH}_{3}\right), 3.87\left(6 \mathrm{H}, \mathrm{s}, 3^{\prime}-\mathrm{OCH}_{3}\right.$ and $\left.5^{\prime}-\mathrm{OCH}_{3}\right), 3.84(3 \mathrm{H}, \mathrm{s}$, $\left.4^{\prime}-\mathrm{OCH}_{3}\right) ;{ }^{13} \mathrm{C}$ NMR $\left(100 \mathrm{MHz}, \mathrm{CDCl}_{3}\right) 194.0\left(\mathrm{C}-\beta^{\prime}\right), 155.2\left(\mathrm{C}-2^{\prime}\right)$, 153.8 (C-4'), 151.2 (C-6'), $144.4(\mathrm{C}-\beta), 138.7\left(\mathrm{C}-5^{\prime}\right), 137.5$ (C-3'), 135.4 (C-1), 130.7 (C-4), 129.2 (C-3 and C-5), 128.7 (C-2 and C-6),

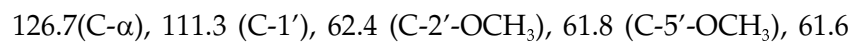
$\left(\mathrm{C}-3^{\prime}-\mathrm{OCH}_{3}\right), 61.3\left(\mathrm{C}-4^{\prime}-\mathrm{OCH}_{3}\right)$; HRESIMS $m / z[\mathrm{M}+\mathrm{H}]^{+} 345.1305$, (calculated for $\mathrm{C}_{19} \mathrm{H}_{21} \mathrm{O}_{6}, 345.1338$ ).

\section{Cell Culture}

The human breast cancer cell line MCF-7 was obtained from American Type Culture Collection (ATCC, USA). MCF-7 cells were cultured in DMEM medium (Welgene Inc., Korea) supplemented with heat-inactivated $10 \%(\mathrm{v} / \mathrm{v})$ fetal bovine serum (FBS; Hyclone Laboratories, USA), and were cultured at $37^{\circ} \mathrm{C}$ in a humidified incubator containing $5 \% \mathrm{CO}_{2}$. 


\section{Assessment of Cell Morphology}

Cells were seeded in a 6-well plate at a density of $3.0 \times 10^{5}$ cells/well and incubated at $37^{\circ} \mathrm{C}$ overnight. After $24 \mathrm{~h}$ of incubation, various concentrations of kanakugiol were added to the cells, and they were incubated further for $24 \mathrm{~h}$. Cell morphology was determined using an inverted phase-contrast microscope (Primovert; Zeiss, Germany).

\section{Cell Viability Assays}

Cell viability was estimated using the 3-(4,5-dimethylthiazol-2-yl)5-(3-carboxy methoxy phenyl)-2-(4-sulfophenyl)-2H-tetrazolium (MTS) assay. The MTS assay is a colorimetric assay used for quantifying the activity of mitochondrial reductase, which reduces the tetrazolium compound to formazan. MCF-7 cells $\left(3 \times 10^{4}\right.$ cells/well) were seeded in 96-well plates containing $100 \mu \mathrm{l}$ of the medium. They were then treated with various concentrations of kanakugiol. The effect of kanakugiol on cell viability was estimated using the CellTiter 96 AQueous One Solution Assay (Promega, USA), containing MTS and phenazine methosulfate, an electron-coupling reagent. An aliquot $(20 \mu \mathrm{l})$ of the aqueous solution of the One reagent was added to each well, followed by incubation for another $1 \mathrm{~h}$. The absorbance of the samples was measured at $492 \mathrm{~nm}$ using a microplate reader (Apollo LB 9110, Berthold Technologies GmbH, Germany). The percentage of viable cells was normalized relative to the untreated controls, and represented as the cell viability $(\%)=\left(\frac{O D \text { of treated }}{\text { OD of control }}\right) \times$ 100. The viability assay was repeated three times.

\section{Annexin-Propidium Iodide (PI) Staining}

Cells $\left(3 \times 10^{5}\right.$ cells/well) were seeded into a 6-well plate and incubated for $24 \mathrm{~h}$. Then, the cells were treated with kanakugiol in a dose-dependent manner and incubated at $37^{\circ} \mathrm{C}$ for a further $24 \mathrm{~h}$. The cells were then washed and harvested using trypsinEDTA. Finally, the cells were collected, and stained using Annexin V-fluorescein isothiocynate (FITC) Apoptosis Detection Kit (BD Bioscience, USA) for $15 \mathrm{~min}$. The proportion of apoptotic cells was determined by using a NovoCyte Flow Cytometer (ACEA Biosciences, USA), and performing analysis using NovoExpress software (ACEA Biosciences).

\section{DNA Laddering}

MCF-7 cells $\left(9 \times 10^{5}\right.$ cells/well) were treated with doxorubicin or kanakugiol for $24 \mathrm{~h}$, washed with ice-cold phosphate-buffered saline (PBS, pH 7.4), and harvested using trypsin-EDTA. The gDNA products were obtained using a G-spin Genomic DNA Extraction Kit according to the manufacturer's instructions (iNtRON Biotechnology, Korea). DNA was loaded on 1.5\% agarose gel and detected by electrophoresis.

\section{Reverse Transcription-PCR}

Cells were lysed in $1 \mathrm{ml}$ of solution with a easy-BLUE Total RNA Extraction Kit (iNtRON Biotechnology), and RNA was isolated according to the manufacturer's instructions. Oligo (dT)primed RNA $(5 \mu \mathrm{g})$ was reverse transcribed using M-MuLV reverse transcriptase (New England Biolabs, USA). Reverse transcription polymerase chain reaction (RT-PCR) was performed on a PCR Thermal Cycler Dice (Takara Bio Inc., Japan) by using the following primer sets: DR3 F 5 '- CAG ATG TTC TGG GTC CAG GT-3' and DR3 R 5'-GCT GTC CAA GGG TGA CAG AT-3', FADD F 5'-CAC AGA CCA CCT GCT TCT GA-3' and FADD R 5'CTG GAC ACG GTT CCA ACT TT-3', Fas F 5'- ATA AGC CCT GTC CTC CAG GT-3', Fas R 5'- TGG AAG AAA AAT GGG CTT TG-3', TRAIL F 5'-GGA ACC CAA GGT GGG TAG AT-3', TRAIL R 5'-TCT CAC ACT GCA ACC TC-3', and GAPDH F 5'-GAG TCA ACG GAT TTG GTC GT-3', GAPDH R 5'-GAC AAG CTT CCC GTT CTC AG-3'.

\section{Western Blotting}

Cells were harvested using trypsin-EDTA and lysed in RIPA buffer (iNtRon Biotechnology, ) at $4^{\circ} \mathrm{C}$ for $2 \mathrm{~h}$. Cell lysates were clarified by centrifugation at $17,010 \times g$ and $4^{\circ} \mathrm{C}$ for $30 \mathrm{~min}$. Protein concentrations were estimated using the Bradford assay (Bio-Rad, USA) and a UV/VIS spectrophotometer (Biowave; Biochrom, UK). Equal amounts of cell lysates were resolved by performing sodium dodecyl sulphate-polyacrylamide gel electrophoresis on $10-15 \%$ gels. Protein bands obtained were transferred onto polyvinylidene difluoride membranes (Cat No: IPVH00010; Millipore, USA). The membranes were blocked with Tris-buffered saline containing Tween-20 [TBST; $2.7 \mathrm{M} \mathrm{NaCl}, 54 \mathrm{mM} \mathrm{KCl}, 1 \mathrm{M}$ Tris- $\mathrm{HCl}$ (pH 7.4), and 0.1\% Tween-20], and 5\% non-fat dried milk for $1 \mathrm{~h}$ at room temperature. Next, the membranes were incubated overnight at $4^{\circ} \mathrm{C}$ with primary antibodies (diluted 1:1000) specific to each target protein. After washing three times with TBST, the membranes were incubated with horseradish peroxidaseconjugated anti-rabbit or anti-mouse IgG secondary antibodies for $1 \mathrm{~h}$ at room temperature. After washing three times with TBST, the blots were detected using a WesternBright ECL Western Blotting Detection Kit (Advansta, USA). The following primary human monoclonal antibodies were used: poly (ADP-ribose) polymerase (PARP) 9542s, caspase-9 9052s, caspase-3 9662s, Bax 2772s, Bid 2002s, Bcl-2 2876s, Bcl-xL 2762s (all purchased from Cell Signaling Technology, USA), glyceraldehyde-3-phosphate dehydrogenase (GAPDH) sc-25778 (purchased from Santa Cruz Biotechnology, USA).

\section{Analysis of Mitochondrial Membrane Potential (MMP)}

MMP $(\Delta \psi \mathrm{m})$ was evaluated via JC-1 staining and flow cytometry. MCF-7 cells $\left(9 \times 10^{5}\right.$ cells/well $)$ were seeded into $3 \mathrm{ml}$ medium in a $60 \mathrm{~mm}$ culture dish and treated with various concentrations of kanakugiol. The cells were harvested using trypsin-EDTA and transferred into $1.5 \mathrm{ml}$ tubes. JC-1 $(5 \mu \mathrm{g} / \mathrm{ml})$ was added to the tube, and mixed until it was completely dissolved. Subsequently, the cells were incubated in the dark for $10 \mathrm{~min}$ at $37^{\circ} \mathrm{C}$, centrifuged $\left(300 \times g, 5 \mathrm{~min}, 4^{\circ} \mathrm{C}\right)$, washed twice 
with PBS, and resuspended in $200 \mu$ PBS. The solutions were protected from light and analyzed by using NovoCyte Flow Cytometer (ACEA Biosciences, USA) and NovoExpress software (ACEA Biosciences).

\section{Cell Cycle Analysis}

Approximately, $3 \times 10^{5}$ cells/well were seeded into 6-well plates and incubated overnight. The cells were treated with different concentrations of kanakugiol and incubated further for $24 \mathrm{~h}$. They were then washed, harvested, and fixed with ice-cold $70 \%$ ethanol at $20^{\circ} \mathrm{C}$. After fixation, the cells were washed with PBS, and then stained with PBS containing $50 \mu \mathrm{g} / \mathrm{ml} \mathrm{PI}$ (Propidium iodide) and $100 \mu \mathrm{g} / \mathrm{ml} \mathrm{RNase} \mathrm{A} \mathrm{for} 30 \mathrm{~min}$. The proportion of apoptotic cells was determined by performing flow cytometry analysis using a FACSCalibur device (BD Biosciences, USA), and CellQuest software (BD Biosciences).

\section{Immunofluorescence Staining}

MCF-7 cells $\left(3 \times 10^{5}\right.$ cells/well) were seeded into a 6-well plate, and incubated at $37^{\circ} \mathrm{C}$ overnight. The cells were then washed twice with PBS and treated with kanakugiol for $24 \mathrm{~h}$. After treatment, the cells were fixed and permeabilized using $4 \%$ formaldehyde for $15 \mathrm{~min}$ at room temperature. Nonspecific sites were blocked by treatment with $1 \%$ bovine serum albumin (BSA) in PBS for $1 \mathrm{~h}$ at room temperature. Then, the cells were incubated overnight at $4^{\circ} \mathrm{C}$ with a mouse polyclonal primary antibody against $\alpha$-tubulin (Santa Cruz Biotechnology) diluted 1:50 in PBS containing $0.1 \%$ BSA and washed three times with PBS (5 min/ wash). Normal mouse IgG antibody was used as control. Next, the cells were incubated with FITC-labelled goat anti-mouse IgG secondary antibody (Merck Millipore, Germany) diluted 1:200 in PBS containing $0.1 \%$ BSA for $1 \mathrm{~h}$ at room temperature. After washing twice with PBS, the cells were stained with 4,6diamidino-2-phenylindole (DAPI; Sigma-Aldrich, USA) for $10 \mathrm{~s}$ at room temperature. Fluorescence images were obtained using an BX61-32FDIC upright fluorescence microscope (Olympus, Japan) equipped with the $100 \times$ objective lens.

\section{Statistical Analyses}

One-way analysis of variance (ANOVA) was conducted. Three independent experiments were performed. The data are expressed as mean \pm standard deviation (SD, $n=3$ ). A $p$-value $<0.05$ was considered statistically significant.

\section{Results and Discussion}

\section{Kanakugiol Reduced Breast Cancer Cell Viability}

Free radical-scavenging and antifungal activities of kanakugiol have been previously reported $[19,20]$. Based on these findings, we investigated the potential anti-cancer effects of kanakugiol (Fig. 1A). For this purpose, we quantified the effects of kanakugiol on the growth of human MCF-7 breast cancer cells. MCF-7 breast cancer cells were treated with various concentrations of kanakugiol for different time periods. Optical microscopy analyses showed

A<smiles>COc1c(O)c(C(=O)/C=C/c2ccccc2)c(OC)c(OC)c1OC</smiles>

B

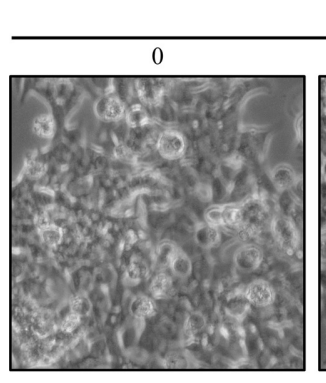

Kanakugiol $(\mu \mathrm{M})$

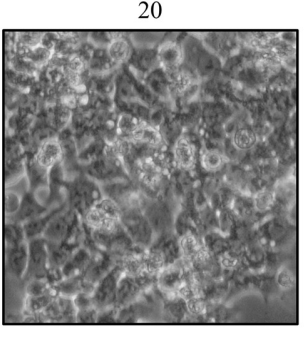

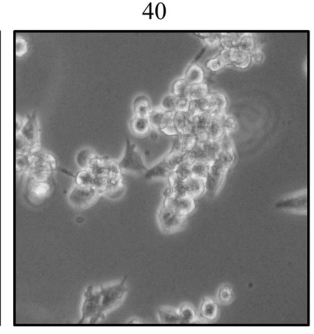

C

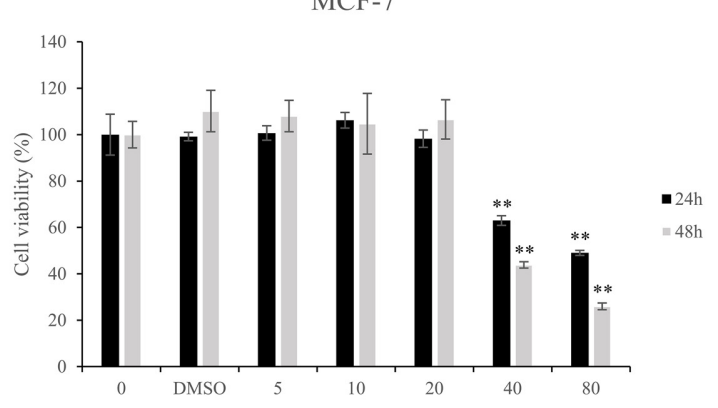

Fig. 1. The inhibitory effect of kanakugiol on the viability of MCF-7 breast cancer cells.

(A) Chemical structure of kanakugiol. (B) Micrographs of MCF-7 cells treated with the indicated concentrations of kanakugiol. (C) MCF-7 cells were treated with the indicated concentrations of kanakugiol for $24 \mathrm{~h}$ and cell viability was assessed by the MTS assay. Data are presented as mean \pm standard deviation $(n=3) .{ }^{* *} p<0.005$ versus control cells. 
a decrease in the proportion of viable MCF-7 cells upon kanakugiol treatment (Fig. 1B). We also determined the effect of kanakugiol on MCF-7 cell viability using the MTS assay. The viability of MCF-7 breast cancer cells decreased in a dose- and time-dependent manner upon kanakugiol treatment (Fig. 1C). Our data thus suggest that kanakugiolmediated MCF-7 cell death.

\section{Kanakugiol Induced Cell Death by Mediating Necrosis with Partial Intrinsic Apoptotic Pathways}

We next sought to clarify whether the kanakugiolmediated cell death was due to apoptosis. Apoptosis is a cell death process that is important in maintaining the balance of cell death and proliferation [21]. Upon induction of apoptosis, phosphatidylserine, a component of the cell membrane, performs a flip-flop transition from the inner to the outer membrane [22]. Annexin V-PI staining assay revealed non-significant alterations in early $(1.23 \%)$ and late apoptosis $(5.11 \%)$ levels in kanakugiol-treated cells.
However, necrosis levels increased dramatically (31.82\%) (Fig. 2A).

Doxorubicin induces apoptosis by activating a variety of apoptotic signals [23]. DNA fragmentation is one of the most specific features of apoptosis. Here, a DNA laddering pattern is formed due to internucleosomal DNA cleavage [24]. Unlike kanakugiol, doxorubicin induced DNA laddering (Fig. 2B). Furthermore, kanakugiol treatment caused an increase in the levels of cleaved PARP (50 kDa), a major PARP fragment observed in necrotic cell death [16].

The intrinsic and extrinsic pathways are the two types of apoptotic pathways. Mitochondrial functions are closely associated with the intrinsic pathway [25]. The family of Bcl-2 proteins is an important component of apoptotic pathway. The main function of Bcl-2 family members is to regulate apoptosis [26]. The Bcl-2 protein family includes two groups: anti-apoptotic Bcl-2, Bcl-xL and pro-apoptotic Bid, Bax, and Bad proteins [27]. We investigated the effect of kanakugiol on factors related to the mitochondrial

A

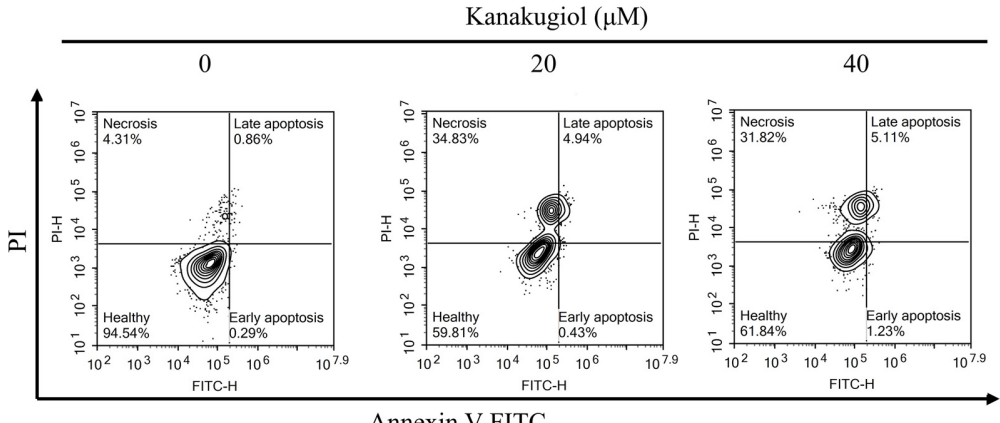

B

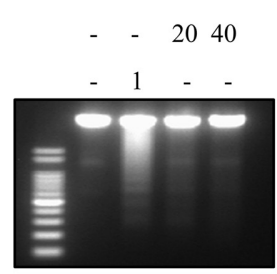

C

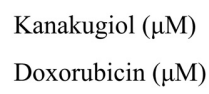

Doxorubicin $(\mu \mathrm{M})$

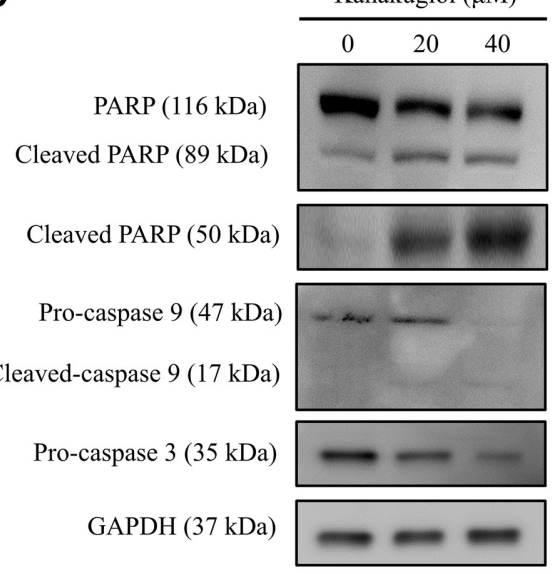

D

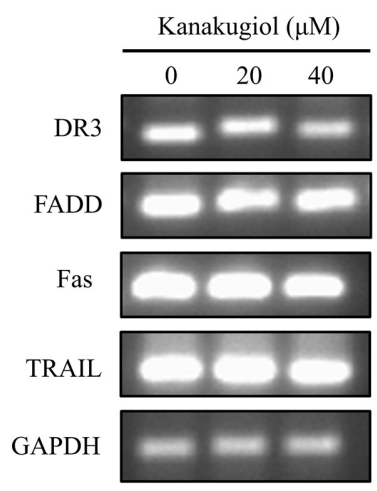

Fig. 2. The effects of kanakugiol on necrosis and apoptosis in MCF-7 breast cancer cells.

(A) MCF-7 cells were treated with varying doses of kanakugiol for $24 \mathrm{~h}$. Apoptosis/necrosis was detected by a flow cytometer using Annexin-V/ PI staining as described in Methods section. (B) MCF-7 cells were treated with doxorubicin or kanakugiol for $24 \mathrm{~h}$ and the gDNA products were loaded onto 1.5\% agarose gel. DNA fragmentation was not induced by kanakugiol-treated MCF-7 cells. (C) Western blot analyses to determine the expression levels of the intrinsic apoptotic markers. (D) Expression levels of death receptors as detected by PCR. 
A

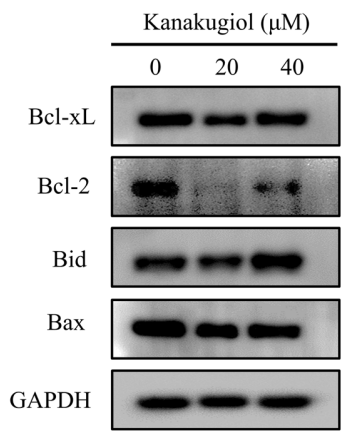

\section{B}

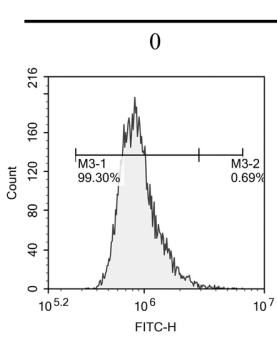

Kanakugiol $(\mu \mathrm{M})$

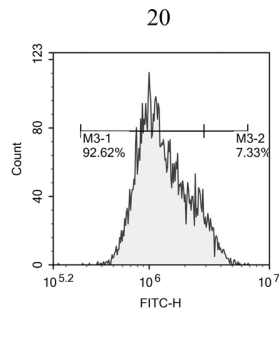

Fluorescence

Fig. 3. Effects of kanakugiol on factors involved in the function of mitochondria in MCF-7 breast cancer cells.

(A) Western blots of mitochondrial Bcl-2 family in MCF-7 cells. (B) Kanakugiol induced mitochondrial membrane potential collapse. The difference in JC-1 colors was analyzed by flow cytometry. intrinsic pathway. Bcl-2 expression was suppressed (Fig. 3A). The cleavage of apoptotic factors was slightly enhanced, and mitochondrial membrane potential collapsed in kanakugiol-treated MCF-7 cells (Figs. 2C and 3B). The levels of death receptor family factors, which are associated with the extrinsic apoptotic pathway [27], remained unaffected in kanakugiol-treated cells (Fig. 2D).

Necroptosis, one mode of programmed necrosis, is a planned and genetically programmed type of cell death. RIPK1, RIPK3 (Receptor interacting kinase 1,3) and MLKL (Mixed lineage kinase domain-like pseudokinase) are necroptosis factors that play significant roles in necroptosis pathway [28]. To investigate whether kanakugiol-induced necrosis type is generalized necrosis or necroptosis, we checked the mRNA expressions of the factors by RT-PCR. The data showed no increase in the levels of the factors (Fig. 4S); therefore, kanakugiol-induced necrosis type is generalized necrosis, not necroptosis. Taken together, cell death was induced via necrosis with partial mitochondrial intrinsic apoptotic pathways upon kanakugiol treatment in MCF-7 cells.

\section{Kanakugiol Induced Mitotic Catastrophe after Cell Cycle Arrest in MCF-7 Cells}

Cell death and proliferation are closely associated with cell cycle [29]. The specific cell cycle phase can be identified

A

Kanakugiol $(\mu \mathrm{M})$

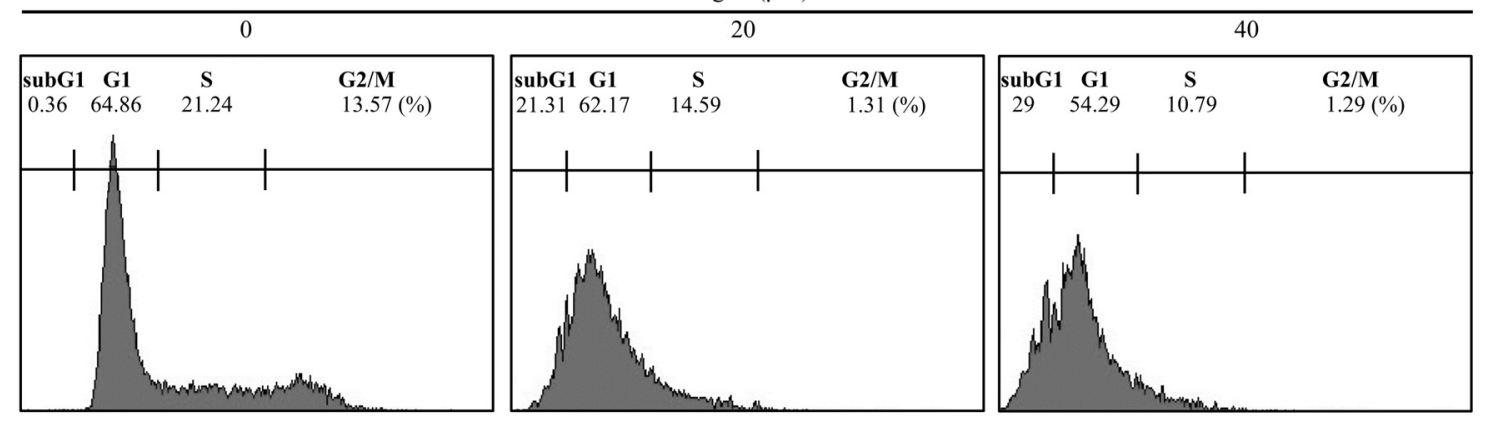

B

Control

Kanakugiol $(40 \mu \mathrm{M})$
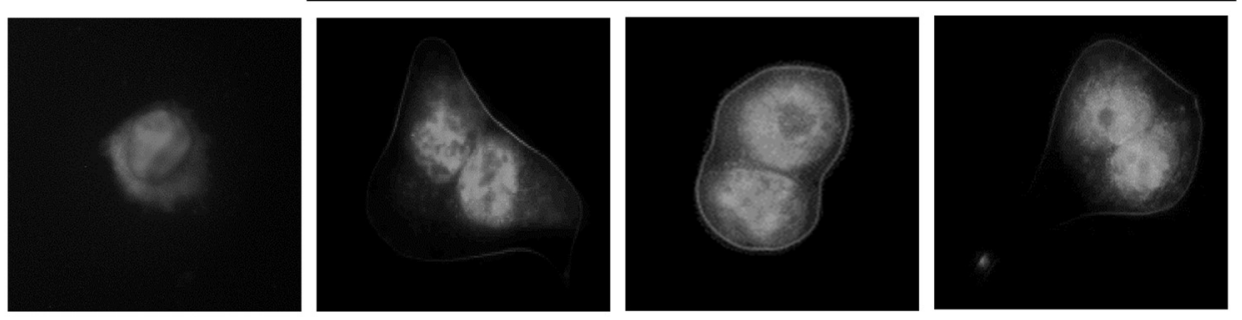

Fig. 4. Effects of kanakugiol on cell cycle progression and mitotic catastrophe (MC) in MCF-7 breast cancer cells.

(A) Cell cycle analysis by FACS using PI-staining. (B) Images of nuclear morphology and microtubule filaments were determined by performing DAPI and $\alpha$-tubulin staining in kanakugiol-treated MCF-7 cells. Micrographs were taken using a fluorescence microscope (magnification, 100×). 


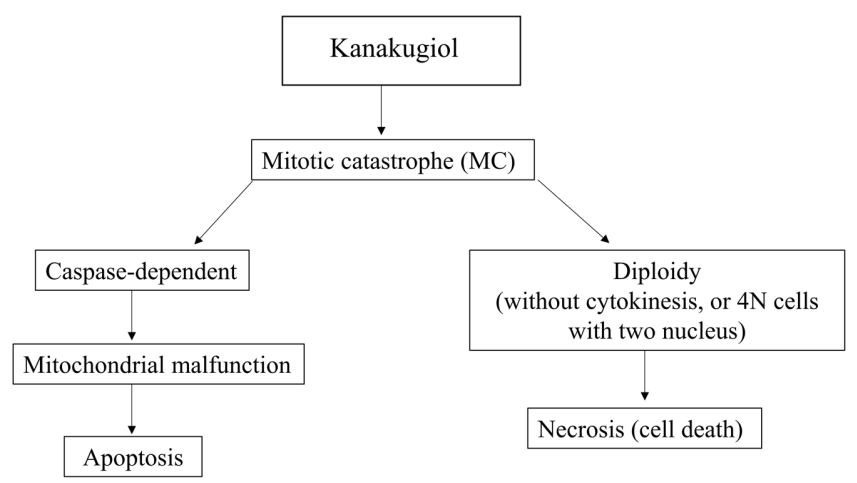

Fig. 5. Mimetic diagram of kanakugiol efficacy pathway in MCF-7 cells.

Kanakugiol inhibits proliferation of MCF-7 cells through mitotic catastrophe. Kanakugiol can induce major necrosis and minor apoptosis.

by flow cytometry which precisely quantifies the cellular DNA content. DNA fragmentation is one of the typical features of apoptotic cells, and PI-staining assay is commonly used for cell death detection [30]. Cell cycle analysis demonstrated that kanakugiol induced cell cycle arrest in sub-G1 phase in MCF-7 cells (Fig. 4A). The proportion of cells in sub- $G_{1}$ phases increased upon kanakugiol treatment. Mitotic catastrophe denotes cell death due to delayed mitosis. In order to obtain more direct evidence regarding mitotic catastrophe, we performed an immunofluorescence assay for staining $\alpha$-tubulin. Immunofluorescence results showed the abnormal structures of microtubules and diploidy in kanakugiol-treated cells (Fig. 4B). Taken together, kanakugiol induced mitotic exit without cytokinesis (4N cells with two nuclei), suggesting that mitotic catastrophe may have been induced via microtubule destabilization. Furthermore, mitotic catastrophe may also have been induced after cell cycle arrest in MCF-7 cells. These findings suggest that kanakugiol inhibits cell proliferation and promotes cell death by inducing mitotic catastrophe after cell cycle arrest. Thus, kanakugiol shows potential for use as a drug in human breast cancer treatment. A model summarizing the effects of kanakugiol is presented in Fig. 5.

\section{Acknowledgements}

This paper was supported by a National Research Foundation of Korea (NRF) grant funded by the Korean Government (2018R1A2B2001225) and was written as part of Konkuk University's research support program for its faculty on sabbatical leave in 2019.

\section{Conflict of Interest}

The authors have no financial conflicts of interest to declare.

\section{Nomenclature}

PARP: poly ADP-ribose polymerase; FITC/PI: fluorescein isothiocyanate/propidium iodide; MTS; 3-(4,5-dimethylthiazol2-yl)-5-(3-carboxy methoxy phenyl)-2-(4-sulfophenyl)-2Htetrazolium.

\section{References}

1. Phosri S, Jangpromma N, Chang LC, Tan GT, Wongwiwatthananukit S, Maijaroen S, et al. 2018. Siamese crocodile white blood cell extract inhibits cell proliferation and promotes autophagy in multiple cancer cell lines. J. Microbiol. Biotechnol. 28: 1007-1021.

2. Holland AJ, Cleveland DW. 2012. Losing balance: the origin and impact of aneuploidy in cancer. EMBO Rep. 13: 501-514.

3. Gordon DJ, Resio B, Pellman D. 2012. Causes and consequences of aneuploidy in cancer. Nat. Rev. Genet. 13: 189-203.

4. Boveri T. 2008. Concerning the origin of malignant tumours by Theodor Boveri. Translated and annotated by Henry Harris. J. Cell Sci. 121 Suppl 1: 1-84.

5. Yoon JH, Pham TH, Lee J, Lee J, Ryu HW, Oh SR, et al. 2019. Methyl linderone suppresses TPA-stimulated IL-8 and MMP-9 expression via the ERK/STAT3 pathway in MCF-7 breast cancer cells. J. Microbiol. Biotechnol. 10.4014/jmb.1911. 11068.

6. Jones EF, Ray KM, Li W, Seo Y, Franc BL, Chien AJ, et al. 2017. Dedicated breast positron emission tomography for the evaluation of early response to neoadjuvant chemotherapy in breast cancer. Clin. Breast Cancer 17: e155-e159.

7. Howell A, Anderson AS, Clarke RB, Duffy SW, Evans DG, Garcia-Closas M, et al. 2014. Risk determination and prevention of breast cancer. Breast Cancer Res. 16: 446.

8. Gewirtz DA. 2000. Growth arrest and cell death in the breast tumor cell in response to ionizing radiation and chemotherapeutic agents which induce DNA damage. Breast Cancer Res. Treat. 62: 223-235.

9. Morse DL, Gray H, Payne CM, Gillies RJ. 2005. Docetaxel induces cell death through mitotic catastrophe in human breast cancer cells. Mol. Cancer Ther. 4: 1495-1504.

10. Mohammad RM, Muqbil I, Lowe L, Yedjou C, Hsu HY, Lin LT, et al. 2015. Broad targeting of resistance to apoptosis in cancer. Semin. Cancer Biol. 35 Suppl: S78-S103.

11. Dumontet C, Jordan MA. 2010. Microtubule-binding agents: a dynamic field of cancer therapeutics. Nat. Rev. Drug Discov. 9: 790-803. 
12. Vitale I, Galluzzi L, Castedo M, Kroemer G. 2011. Mitotic catastrophe: a mechanism for avoiding genomic instability. Nat. Rev. Mol. Cell Biol. 12: 385-392.

13. Ali AG, Mohamed MF, Abdelhamid AO, Mohamed MS. 2017. A novel adamantane thiadiazole derivative induces mitochondria-mediated apoptosis in lung carcinoma cell line. Bioorg. Med. Chem. 25: 241-253.

14. Moubarak RS, Yuste VJ, Artus C, Bouharrour A, Greer PA, Menissier-de Murcia J, et al. 2007. Sequential activation of poly(ADP-ribose) polymerase 1, calpains, and Bax is essential in apoptosis-inducing factor-mediated programmed necrosis. Mol. Cell Biol. 27: 4844-4862.

15. Nicotera P, Lipton SA. 1999. Excitotoxins in neuronal apoptosis and necrosis. J. Cereb. Blood Flow Metab. 19: 583-591.

16. Shah GM, Shah RG, Poirier GG. 1996. Different cleavage pattern for poly(ADP-ribose) polymerase during necrosis and apoptosis in HL-60 cells. Biochem. Biophys. Res. Commun. 229: 838-844.

17. Mc Gee MM. 2015. Targeting the mitotic catastrophe signaling pathway in cancer. Mediators Inflamm. 2015: 146282.

18. Galluzzi L, Vitale I, Abrams JM, Alnemri ES, Baehrecke EH, Blagosklonny MV, et al. 2012. Molecular definitions of cell death subroutines: recommendations of the nomenclature committee on cell death 2012. Cell Death Differ. 19: 107-120.

19. Fan H, Zheng T, Chen Y, Yang GZ. 2012. Chemical constituents with free-radical-scavenging activities from the stem of Fissistigma polyanthum. Pharmacogn. Mag. 8: 98-102.

20. Hwang EI, Lee YM, Lee SM, Yeo WH, Moon JS, Kang TH, et al. 2007. Inhibition of chitin synthase 2 and antifungal activity of lignans from the stem bark of Lindera erythrocarpa. Planta Med. 73: 679-682.
21. Zhao YR, Li HM, Zhu M, Li J, Ma T, Huo Q, et al. 2018. Non-benzoquinone geldanamycin analog, WK-88-1, induces apoptosis in human breast cancer cell lines. J. Microbiol. Biotechnol. 28: 542-550.

22. Marino G, Kroemer G. 2013. Mechanisms of apoptotic phosphatidylserine exposure. Cell Res. 23: 1247-1248.

23. Tacar O, Sriamornsak P, Dass CR. 2013. Doxorubicin: an update on anticancer molecular action, toxicity and novel drug delivery systems. J. Pharm. Pharmacol. 65: 157-170.

24. Majtnerova P, Rousar T. 2018. An overview of apoptosis assays detecting DNA fragmentation. Mol. Biol. Rep. 45: 1469-1478.

25. Fulda S, Debatin KM. 2006. Extrinsic versus intrinsic apoptosis pathways in anticancer chemotherapy. Oncogene 25: 47984811.

26. Siddiqui WA, Ahad A, Ahsan H. 2015. The mystery of BCL2 family: Bcl-2 proteins and apoptosis: an update. Arch. Toxicol. 89: 289-317.

27. Taylor RC, Cullen SP, Martin SJ. 2008. Apoptosis: controlled demolition at the cellular level. Nat. Rev. Mol. Cell Biol. 9: 231-241.

28. Thakur B, Kumar Y, Bhatia A. 2019. Programmed necrosis and its role in management of breast cancer. Pathol. Res. Pract. 215: 152652.

29. Pucci B, Kasten M, Giordano A. 2000. Cell cycle and apoptosis. Neoplasia 2: 291-299.

30. Riccardi C, Nicoletti I. 2006. Analysis of apoptosis by propidium iodide staining and flow cytometry. Nat. Protoc. 1: 1458-1461. 